\title{
The Association between Treatment with Bisphosphonates and the Risk of Atrial Fibrillation: A Meta-Analysis of Observational Studies
}

\author{
Ji-Hyun Park, Hae-Jin Ko* \\ Department of Family Medicine, School of Medicine, Kyungpook National University, Kyungpook National University Hospital, Daegu, Korea
}

Background: Osteoporosis is one of the most common diseases of the skeletal system, particularly occurring in older adults. Bisphosphonates are frequently used to treat osteoporosis and prevent bone fractures. Studies evaluating the association between treatment with bisphosphonate and the risk of atrial fibrillation have reported conflicting results. This meta-analysis of observational studies was performed to assess this association.

Methods: Databases were searched to find relevant observational studies, and the identified articles were selected according to the selection criteria. Sensitivity and subgroup analysis based on various confounding factors were performed. The pooled odds ratios (ORs) and 95\% confidence intervals (CIs) for the risk of atrial fibrillation were estimated using a random-effects model.

Results: We selected 12 studies, including four case-control and eight cohort studies, for the meta-analysis. Assessment of the estimated effect size yielded an OR of 1.171 (95\% CI, 1.011-1.356; $\mathrm{P}=0.035$ ), with substantial heterogeneity $\left(\mathrm{I}^{2}=84.74 \%, \mathrm{P}<0.001\right)$. When the studies were excluded one-after-another, the pooled OR remained unchanged in only six studies. In addition, subgroup analyses found that treatment with bisphosphonates was positively associated with the risk of atrial fibrillation in studies performed in Western countries (OR, 1.263; 95\% CI, 1.092-1.462) and lower-quality studies (OR, 1.214; 95\% CI, 1.035-1.423). No publication bias was observed.

Conclusion: This meta-analysis showed that treatment with bisphosphonates may be associated with an increased risk of atrial fibrillation. Therefore, bisphosphonates should be carefully prescribed to patients at a high risk of atrial fibrillation.

Keywords: Atrial Fibrillation; Bisphosphonates; Alendronate; Risedronic Acid; Ibandronic Acid; Zoledronic Acid

Received: June 17, 2021, Revised: July 27, 2021, Accepted: August 14, 2021

*Corresponding Author: Hae-Jin Ko https://orcid.org/0000-0003-4460-1476

Tel: +82-53-200-6578, Fax: +82-53-200-5480, E-mail: liveforme@knu.ac.kr 


\section{INTRODUCTION}

Osteoporosis is one of the most common diseases of the skeletal system, particularly occurring in older individuals. ${ }^{1)}$ According to the National Osteoporosis Foundation, 54 million adults in the United States, aged $\geq 50$ years, are affected by osteoporosis or low bone mass, and 43 million adults are at a risk of developing osteoporosis. ${ }^{2)}$

Bisphosphonates (BPs) are a class of drugs most frequently prescribed to treat osteoporosis and prevent bone fracture. ${ }^{3-5)}$ BPs suppress bone turnover, prevent bone loss, preserve bone architecture by tightly binding to the bone, and are internalized by osteoclasts to inhibit bone resorption. ${ }^{6,7)}$ Four BPs, alendronate, risedronate, ibandronate, and zoledronate, have been approved for the treatment and prevention of osteoporosis in Korea. ${ }^{3,6)}$ Although BPs are considered firstline treatments for osteoporosis, they are associated with various side effects, including gastrointestinal disorders, renal toxicity, flu-like symptoms with fever, ocular events, and, more rarely, osteonecrosis of the jaw and atrial fibrillation (AF) ${ }^{8,9)}$ Studies evaluating the association between treatment with BPs and an increased risk of AF have yielded conflicting results, making the association between BPs with AF uncertain. $^{3-5,7,10-17)}$

Globally, AF is one of the most common clinical arrhythmias, especially in older persons. ${ }^{18,19)}$ The Framingham Heart Study reported that the prevalence of AF has increased by three-folds over the past 50 years. ${ }^{20)}$ In addition to age, AF has many risk factors, including hypertension, diabetes mellitus, heart failure, coronary heart disease, chronic obstructive pulmonary disease, and chronic kidney disease. Environmental risk factors for AF include obesity, physical inactivity, alcohol consumption, and smoking. ${ }^{5,18,21)} \mathrm{AF}$ is a leading cause of morbidity and is associated with a poor quality of life, heart failure, myocardial infarction, dementia, stroke, and death. ${ }^{18,19,22)}$ The incidence and overall prevalence of AF increases with age, making AF a major concern. ${ }^{19,23,24)}$ The prevalence of osteoporosis also increases with age, and so does the requirement of treatment with BPs, suggesting a need to evaluate whether the use of BPs is associated with a greater risk of $\mathrm{AF}^{1,12,25)}$ This meta-analysis of observational studies aimed to evaluate the association between $\mathrm{BP}$ treatment and the risk of AF in patients with osteoporosis.

\section{METHODS}

We searched three databases, CINAHL with Full Text, PubMed, and SCOPUS, for articles concerning the association between treatment with BPs and the risk of AF that were published between January 2007 and July 15, 2020. Search terms included "Bisphosphonates" OR "Alendronate" OR "Risedronate" OR "Ibandronate" OR "Zoledronate" AND "Atrial fibrillation."

The identified publications were independently reviewed by two authors (J.H.P. and H.J.K.) who selected relevant observational studies. Duplicate articles and articles that were not written in English were excluded. The titles and abstracts of articles were reviewed to exclude ir- relevant studies, along with comments, reviews, errata, editorials, letters, and research articles. The full texts of the remaining articles were read, and relevant studies were selected. The references of each selected article were also reviewed to identify other relevant articles. All results were exported to a reference manager (EndNote X9; Clarivate, Philadelphia, PA, USA).

The inclusion criteria were as follows: (1) studies that were performed in humans; (2) observational studies; (3) studies that included patients who required BPs for any indication; (4) studies that reported the association between treatment with BPs and the risk of AF; (5) studies that provided calculable data and effects estimates such as odds ratios (ORs) or hazard ratios with $95 \%$ confidence intervals (CIs), or P-values; and (6) studies that were written in English.

Studies were excluded if they were: (1) duplicated studies; (2) nonobservational studies; (3) in vitro or pre-clinical in vivo studies; (4) comments, reviews, errata, editorials, letters, or abstracts; or (5) containing inadequate data.

The association between treatment with BPs and the risk of AF was evaluated by calculating the pooled OR and 95\% CI using the Dersimonian and Laird random effects model. This method is regarded as a standard approach for meta-analyses of clinical studies, and is considered useful in providing an overall effect estimate across related studies. $^{26)}$

The heterogeneity of the selected studies was assessed by calculating the P-value of the $\chi^{2}$-based Cochrane $\mathrm{Q}$ tests, with a P-value $<0.05$ indicating significant heterogeneity. ${ }^{27)}$ Study heterogeneity was also determined by calculating the inconsistency score $\left(\mathrm{I}^{2}\right)$, which ranged from $0 \%$ (no heterogeneity) to $100 \%$ (maximum heterogeneity), with $\mathrm{I}^{2}$ values of $25 \%, 50 \%$, and $75 \%$ indicating low, moderate, and high heterogeneity, respectively, and $\mathrm{I}^{2}$ values $\geq 50 \%$ considered as significant heterogeneity. ${ }^{27)}$

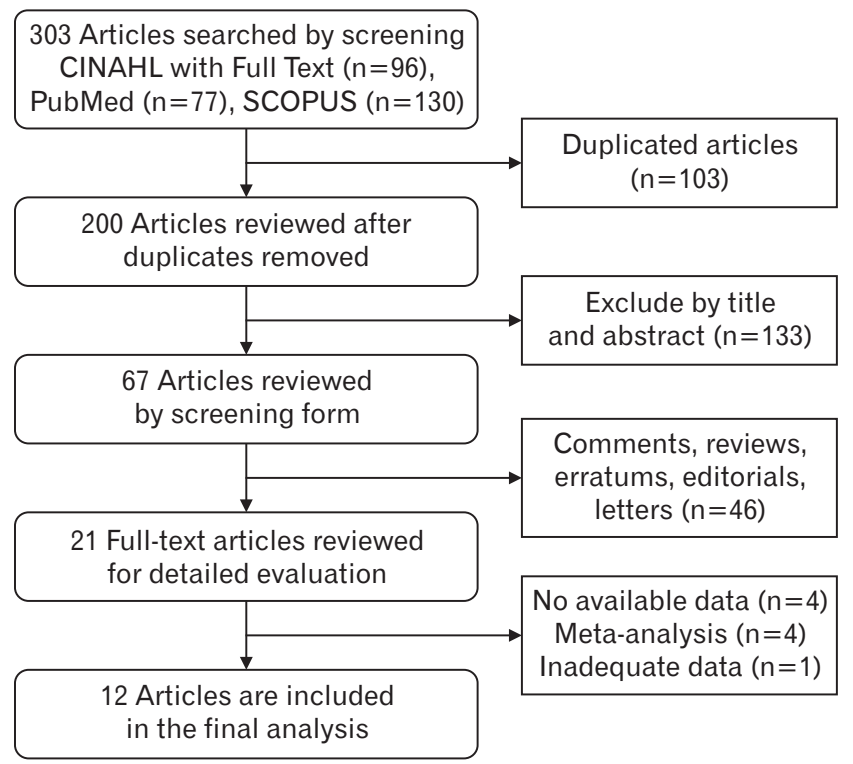

Figure 1. Flow diagram of the process of study selection. 


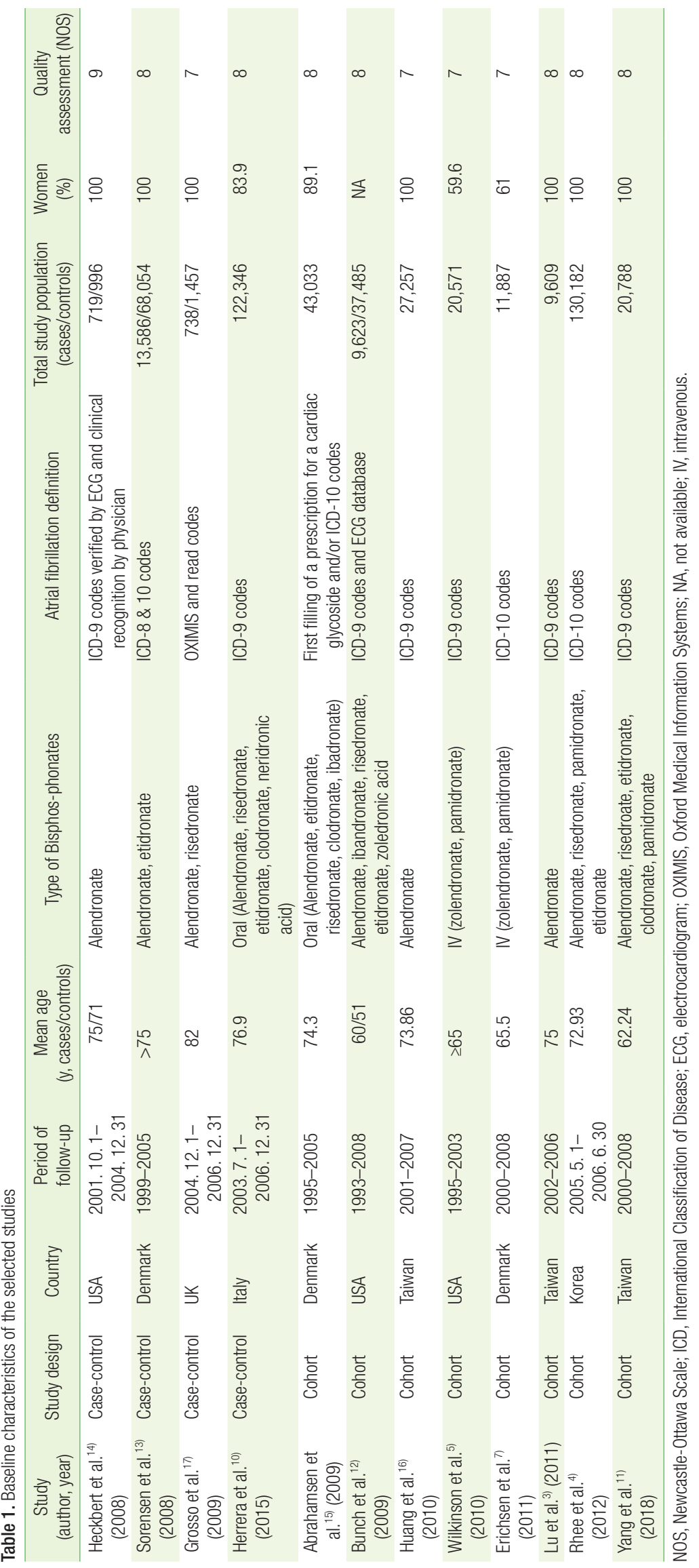


The methodological quality of the studies was assessed using the Newcastle-Ottawa Scale (NOS), ${ }^{28)}$ which included eight criteria, grouped into three parts: selection, comparability, and exposure (casecontrol studies) or outcome (cohort studies). The NOS scores ranged from 0 to 9 , with no definitive cut-off value to identify a high-quality study. In this meta-analysis, the mean NOS value of the selected studies was 7.75. Therefore, studies with an NOS score $>7$ were defined as high-quality.

To estimate the accuracy of the pooled effect size and to supplement the substantial heterogeneity, we conducted a sensitivity analysis by excluding each study individually and evaluating the impact after each study was removed. Subgroup analyses were also performed according to various confounding factors, including study design (case-control versus cohort), countries of publication (Asian versus Western), quality of study (NOS $>7$ versus $\leq 7$ ), and sex (female only).

Potential publication bias was assessed using Begg's funnel plot and rank test. ${ }^{29)}$ The asymmetry of distribution was analyzed in the funnel plot of standard error by determining $\log \mathrm{OR}$, with a P-value $<0.05$ indicating statistically significant publication bias.

All statistical analyses were performed using a random effects model and Comprehensive Meta-Analysis ver. 2.2.064 software (Biostat Inc., Englewood, NJ, USA), with statistical significance set at $\mathrm{P}<0.05$.

\section{RESULTS}

\section{Study Selection}

After searching the CINAHL with Full Text, PubMed, and SCOPUS databases between January 2007 and July 2020, we identified 96, 77, and 130 potentially relevant articles, respectively. After excluding duplicate articles, 200 articles remained, of which 133 were deemed irrelevant and were excluded after their titles and abstracts were screened. An additional 46 articles were found to be comments, reviews, errata, editorials, and letters, and were therefore excluded. A full text review of the 21 remaining articles resulted in the exclusion of nine additional articles: four with no available data; four meta-analyses, including three meta-analyses of randomized controlled trials (RCTs); and one with inadequate data. Finally, 12 articles were selected for analysis. Figure 1 shows the flowchart of the process of study selection.

Of the 12 selected studies, four were case-control studies and eight were cohort studies. They were published between January 1, 2007 and July 15, 2020 in various countries. Three studies were conducted in the United States, ${ }^{5,12,14)}$ three in Denmark, ${ }^{7,13,15)}$ three in Taiwan, ${ }^{3,11,16)}$ one in Korea, ${ }^{4)}$ one in Italy, ${ }^{10)}$ and one in the United Kingdom. ${ }^{17)}$ The 12 studies included 518,331 subjects, with a follow-up duration ranging from 1 to 15 years. The mean age of the study participants ranged from 51 to $>75$ years, and seven studies only included women. ${ }^{3,4,11,13,14,16,17)} \mathrm{BP}$ was administered intravenously in two studies, ${ }^{5,7)}$ orally in seven studies, $^{3,10,13-17)}$ and as a mixture of both in three studies. ${ }^{4,11,12)}$ The diagnosis of AF was based on International Classification of Diseases (ICD)-9 codes in seven studies, ${ }^{3,5,10-12,14,16)}$ ICD-10 codes in four studies, ${ }^{4,7,13,15)}$ and medical information systems in one study. ${ }^{17)}$ Eight studies were of high quality, ${ }^{3,4,10-15)}$ whereas four studies were not. ${ }^{5,7,16,17)}$ Two studies evaluated the risk of $\mathrm{AF}$ associated with treatment with $\mathrm{BPs}$ and raloxifene, ${ }^{3,16)}$ and one study evaluated the risk of AF associated with treatment with BPs, vitamin D, and neither. ${ }^{11)}$ The baseline characteristics of the selected studies are summarized in Table 1.

\section{Treatment with Bisphosphonates and the Risk of Atrial Fibrillation}

A meta-analysis using a random-effects model showed that treatment with BPs was significantly associated with the risk of AF (pooled OR, 1.171; 95\% CI, 1.011-1.356; $\mathrm{P}=0.035$ ), with studies showing substantial heterogeneity $\left(\mathrm{I}^{2}=84.74 \%, \mathrm{P}<0.001\right)$ (Figure 2$)$.

\section{Sensitivity Analysis}

According to the pooled OR of each sensitivity analysis performed by

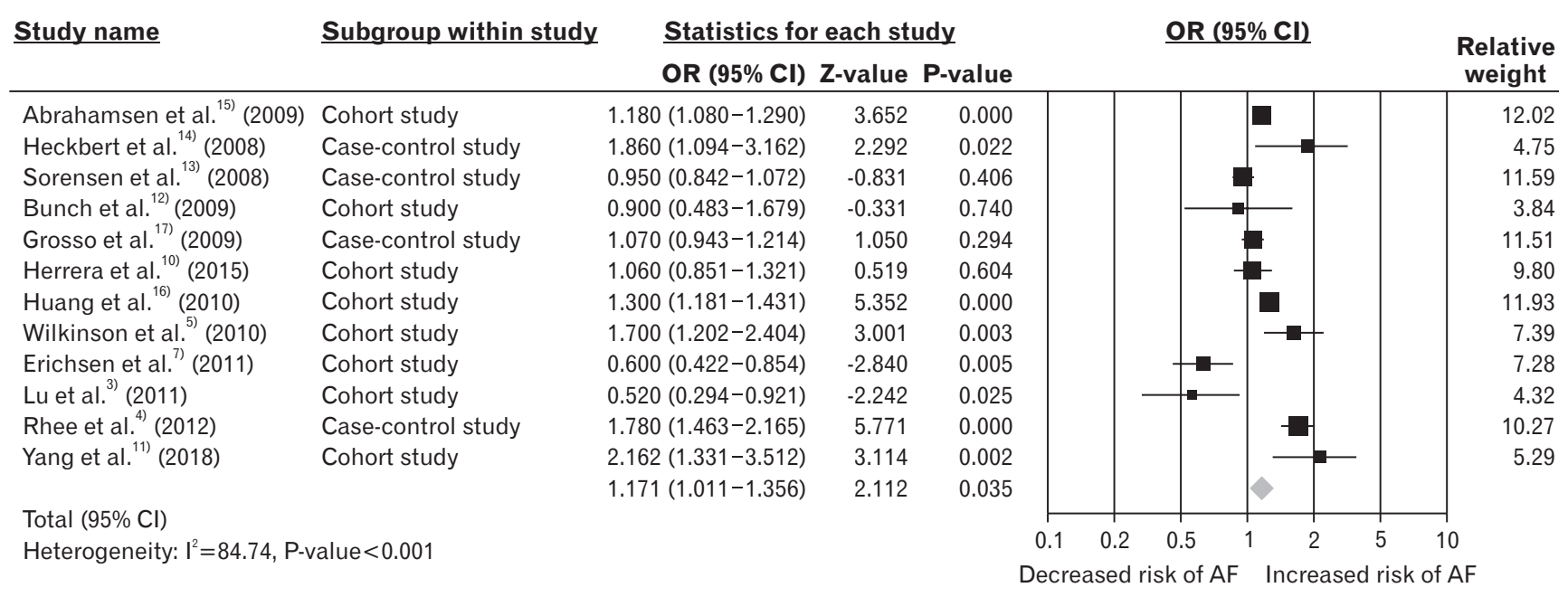

Figure 2. Forest plot of the association between treatment with bisphosphonates and the risk of atrial fibrillation, using a random-effects model. OR, odds ratio; $\mathrm{Cl}$, confidence interval; $A F$, atrial fibrillation. 
excluding each study individually, treatment with BPs was positively associated with the risk of AF in only six studies. There was no significant change in the results of the analysis (Table 2).

\section{Subgroup Analysis}

Subgroup analyses were also performed to assess whether the study design, country of publication, study quality, and female sex had an effect on the association between treatment with BPs and the risk of AF. A positive association between these factors was consistently observed only in studies performed in the Western countries and in lower-quality studies (Table 3 ).

Pooled analysis of the eight studies performed in four Western countries (United States, Denmark, Italy, and the United Kingdom) showed that treatment with BPs was positively associated with the risk of $\mathrm{AF}$ (OR, 1.263; 95\% CI, 1.092-1.462). However, this association was not observed in the pooled analysis of the four studies performed in two Asian countries (Taiwan and Korea: OR, 0.925; 95\% CI, 0.5491.558).

Of the 12 studies included in this meta-analysis, four were case-con-

Table 2. Sensitivity analysis by excluding each study one-after-another

\begin{tabular}{lcc}
\hline & \multicolumn{2}{c}{ Effect size } \\
\cline { 2 - 3 } Excluded study (author, year) & $\mathrm{OR}^{*}(95 \% \mathrm{Cl})$ & P-value \\
\hline Abrahamsen et al. ${ }^{15)}(2009)$ & $1.168(0.975-1.399)$ & 0.091 \\
Heckbert et al. $^{14)}(2008)$ & $1.145(0.986-1.329)$ & 0.076 \\
Sorensen et al..$^{13)}(2008)$ & $1.204(1.030-1.406)$ & 0.019 \\
Bunch et al. ${ }^{12)}(2009)$ & $1.184(1.018-1.376)$ & 0.028 \\
Grosso et al. ${ }^{17)}(2009)$ & $1.184(1.002-1.399)$ & 0.048 \\
Huang et al. ${ }^{6)}(2010)$ & $1.184(1.010-1.387)$ & 0.037 \\
Wilkinson et al. $(2010)$ & $1.154(0.973-1.370)$ & 0.100 \\
Erichsen et al. ${ }^{7)}(2011)$ & $1.137(0.978-1.322)$ & 0.094 \\
Lu et al..$^{3)}(2011)$ & $1.232(1.071-1.418)$ & 0.004 \\
Rhee et al..$^{4)}(2012)$ & $1.213(1.051-1.401)$ & 0.008 \\
Herrera et al. ${ }^{10)}(2015)$ & $1.116(0.970-1.285)$ & 0.126 \\
Yang et al. ${ }^{11)}(2018)$ & $1.133(0.979-1.312)$ & 0.095 \\
\hline
\end{tabular}

$\mathrm{OR}$, odds ratio; $\mathrm{Cl}$, confidence interval.

${ }^{*}$ Mean OR without this study.

Table 3. Subgroup analysis of the association between treatment with bisphosphonates and the risk of atrial fibrillation according to study design, countries, quality of study, and sex, using a random-effects model

\begin{tabular}{|c|c|c|c|}
\hline \multirow{2}{*}{ Subgroup } & \multirow{2}{*}{ No. of studies } & \multicolumn{2}{|l|}{ Effect size } \\
\hline & & $\mathrm{OR}(95 \% \mathrm{Cl})$ & P-value \\
\hline \multicolumn{4}{|l|}{ Study design } \\
\hline Case-control & $4^{13,14,16,17)}$ & $1.290(0.960-1.725)$ & 0.091 \\
\hline Cohort & $8^{3-5,7,10-12,15)}$ & $1.116(0.927-1.343)$ & 0.247 \\
\hline \multicolumn{4}{|l|}{ Countries } \\
\hline Asian & $4^{3,4,11,16)}$ & $0.925(0.549-1.558)$ & 0.769 \\
\hline Western & $8^{5,7,10,12-15,17)}$ & $1.263(1.092-1.462)$ & 0.002 \\
\hline \multicolumn{4}{|l|}{ Quality of study } \\
\hline High (NOS >8) & $8^{3,4,40-15)}$ & $1.125(0.880-1.438)$ & 0.347 \\
\hline Low (NOS $\leq 7)$ & $4^{5,7,76,17)}$ & $1.214(1.035-1.423)$ & 0.017 \\
\hline Women only & $7^{3,4,411,13,14,16,17)}$ & $1.025(0.829-1.267)$ & 0.820 \\
\hline
\end{tabular}

OR, odds ratio; $\mathrm{Cl}$, confidence interval; NOS, Newcastle-Ottawa Scale. trol studies and eight were cohort studies. The association between treatment with BPs and AF was not statistically significant in both the pooled analysis of case-control (OR, 1.290; 95\% CI, 0.960-1.725) and cohort (OR, 1.116; 95\% CI, 0.927-1.343) studies.

The 12 included studies were also grouped based on quality, with eight studies being of high quality (NOS, 8-9) and four of lower quality (NOS, 7). Treatment with BPs was associated with a greater risk of AF in lower-quality studies (OR, 1.214; 95\% CI, 1.035-1.423) but not in high-quality studies (OR, 1.125; 95\% CI, 0.880-1.438).

Of the 12 studies, seven studies only included women. Assessment of this subgroup analysis showed that treatment with BPs was positively associated with the risk of $\mathrm{AF}$ (OR, 1.025; 95\% CI, 0.829-1.267), but the difference was not statistically significant.

\section{Publication Bias}

Publication bias was evaluated using the Begg's rank test and by creating a funnel plot. The funnel plot was symmetrical, with no evidence of obvious asymmetry (Figure 3). The Begg's rank test for asymmetry showed no significant publication bias in this meta-analysis $(\mathrm{P}=0.784)$.

\section{DISCUSSION}

This meta-analysis aimed to clarify the association between the risk of AF and treatment with BPs, which are increasingly used in aging societies around the world. This study is valuable because it evaluates upto-date observational studies performed in various countries. Twelve studies, including 518,331 patients, were analyzed using a random-effects model. To summarize, this meta-analysis showed that treatment with BP was significantly associated with a $17 \%$ greater risk of developing AF. Notably, the results of this meta-analysis of observational studies are consistent with those of a previous meta-analysis that included RCTs, which showed that the risk of AF increased with BP treatment, as compared to the non-BP comparators. ${ }^{30)}$

Although the mechanism by which BP treatment increases the risk

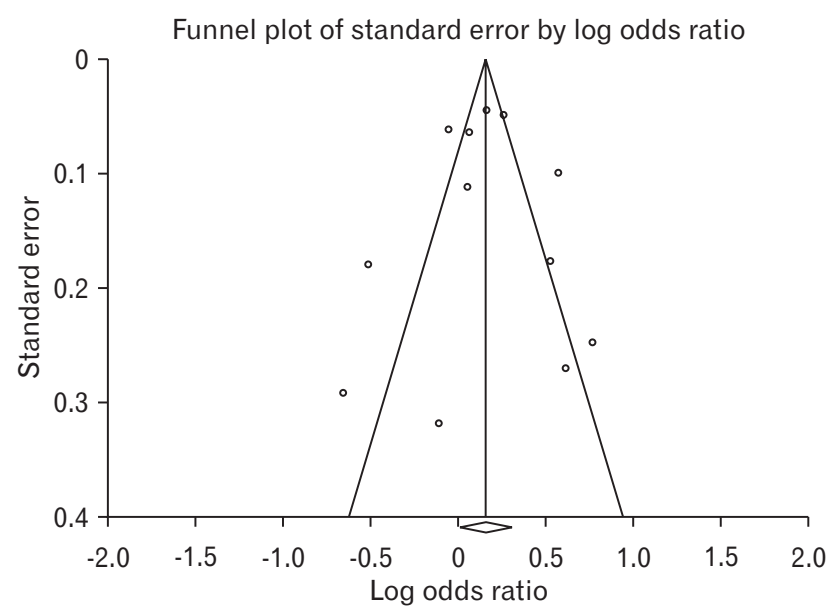

Figure 3. Funnel plot for pulibcation bias. 
of AF remains unclear, several potential mechanisms have been suggested. First, inflammatory cytokines induced by BPs may increase the risk of $\mathrm{AF}^{23,30)}$ Inflammation is a risk factor for $\mathrm{AF}$ and may play a significant role in its initiation. ${ }^{31)} \mathrm{BPs}$, particularly parenteral types, can stimulate the release of inflammatory cytokines, including interleukin-6, C-reactive protein, and tumor necrosis factor- $\alpha .^{32,33)}$ This increase in cytokines can alter atrial conduction and may be responsible for atrial remodeling and fibrosis. ${ }^{10,14)}$ Second, animal studies have shown that BP may affect arterial contraction by being accumulated in the arterial wall. ${ }^{5)}$ Because of their high affinity to calcium and hydroxyapatite, BPs may accumulate in the calcified matrix outside of bones, including in atherosclerotic arteries. ${ }^{8,34)}$ Moreover, BPs may accumulate in an inhomogeneous manner in different histological vessel layers, thereby affecting arterial contraction. However, further studies are needed to clarify this mechanism. ${ }^{8,35)}$ Third, electrolyte imbalance, such as hypocalcemia or hypophosphatemia, can cause $\mathrm{AF}^{13,14,36)} \mathrm{BPs}$, such as calcium, phosphate, zinc, and magnesium, tend to bind to several key electrolytes involved in cardiac conduction. Thus, treatment with BPs may lead to electrolyte imbalance, ultimately causing $\mathrm{AF}^{23,25,30)} \mathrm{BPs}$ can also induce hypocalcemia and hypophosphatemia, altering calcium dynamics in atrial cells by inducing self-sustained calcium oscillations. The atrium is sensitive to reductions in calcium concentration, resulting in irregular depolarization of atrial cells and the induction of $\mathrm{AF}^{25,37)}$ In addition, hypocalcemia and associated secondary hyperparathyroidism caused by treatment with BPs may be responsible for $\mathrm{AF}^{13,25,38)}$ The mechanisms underlying these processes have not yet been clarified, and several hypotheses have been suggested. Fourth, BPs, particularly the intravenous type, can chelate zinc, thereby suppressing zinc-dependent endopeptidases such as matrix metalloproteinases, which promote microvascular growth and are associated with long-term structural changes in the heart, such as collagen deposition and atrial remodeling. ${ }^{23,30}$ Because the effects of BPs on atrial conduction remain unclear, further studies are needed to assess the effects of the dose and duration of treatment with BPs on the occurrence of AF.

Although treatment with BPs is associated with the risk of AF, BPs have benefits in patients with osteoporosis, including the prevention of fractures. ${ }^{2,9)}$ BPs have been found to not only reduce the rates of hip, vertebral, and nonvertebral fractures, but also to reduce morbidity and health care-related costs, and significantly enhance survival rates. ${ }^{9,39)}$ Risk-benefit analysis showed that BPs might have more benefits than risks in elderly patients with osteoporosis and a high risk of fracture, despite the increased risk of AF.

In this meta-analysis, the effect sizes of subgroup analyses based on the type of study design, country of study, study quality, and female sex were not changed. However, only two subgroup analyses, i.e., studies performed in Western countries and lower quality studies, showed a positive association between treatment with BPs and the risk of AF. In the subgroup analysis according to countries, a positive association was observed in studies performed in Western countries, but not in Asian countries. The prevalence of AF is lower in Asian countries as compared to Western countries. ${ }^{19)}$ Moreover, the present meta-analysis included more studies performed in Western countries with a three-fold higher population of Western than Asian patients. These populations differ in the prevalence of risk factors, including genetic and other factors, such as cardiovascular disease and obesity, resulting from lifestyle differences, such as diet, exercise, and other environmental factors. ${ }^{40)}$ Additional studies are needed to assess the effects of geographical location on the association between BP treatment and the risk of AF.

This meta-analysis had several limitations. First, it included only observational studies, which may have introduced biases inherent to the design of these studies, including selection, information, and response biases. ${ }^{41,42)}$ These are innate limitations of meta-analyses, indicating a need for RCTs in large populations and subsequent meta-analyses of the trials. Second, this meta-analysis included studies that were only written in English, therefore, it may have publication bias based on language, although there was no significant publication bias found using Begg's rank test. Third, the studies included in the present metaanalysis showed a high degree of heterogeneity, including differences in the dose and duration of treatment, route of administration, and type of BP used to treat patients. To overcome these limitations, we used a random-effects model to determine the average effect size of these heterogeneous populations and performed sensitivity and subgroup analyses using various factors. Fourth, we did not assess the association between dose or duration of treatment with BPs and the risk of AF, because only four of the 12 included studies analyzed dose responses to BPs, ${ }^{3,5,16,17)}$ and the duration of treatment with BPs was unclear in each study. In addition, the type of BP differed among the included studies, with seven involving treatments with oral administration of BPs, ${ }^{3,10,13-17)}$ two including intravenous administration of BPs, ${ }^{5,7}$ and three including both oral and intravenous administration of BPs. ${ }^{4,11,12)}$ Finally, not all included studies reported other risk factors associated with AF, such as history of cardiovascular disease or diabetes. Although two studies reported history of alcohol use as a potential confounder, ${ }^{2,13)}$ none provided information about other aspects of patient history, including smoking, health behavior, and other potential confounders. Therefore, further evaluation is needed to determine whether the risk of AF is associated with the dose, duration, or type of BP.

Despite these limitations, this is a comprehensive meta-analysis assessing the association between treatment with BPs, most frequently administered to patients with osteoporosis around the world, and the risk of AF. In addition, all meta-analyses utilize a random-effects model, studies were performed in Asian and Western countries, the total study population was large, and patients were treated with all types of BPs prescribed currently in Korea. Finally, a lack of publication bias indicates that this meta-analysis has high reliability.

In conclusion, this meta-analysis of observational studies showed that treatment with BP was significantly associated with an increased risk of AF. BPs are one of the most effective classes of medications used to treat osteoporosis and to prevent related fractures, which are the 
leading cause of mortality, and reduced quality of life among elderly persons. The present findings indicate that BPs should be prescribed with care to patients at a high risk of AF. Therefore, healthcare providers should evaluate the benefits and risks of BPs in individual patients.

\section{CONFLICT OF INTEREST}

No potential conflict of interest relevant to this article was reported.

\section{ORCID}

Ji-Hyun Park: https://orcid.org/0000-0003-2620-6798

Hae-Jin Ko: https://orcid.org/0000-0003-4460-1476

\section{REFERENCES}

1. Choi MH, Yang JH, Seo JS, Kim YJ, Kang SW. Prevalence and diagnosis experience of osteoporosis in postmenopausal women over 50: focusing on socioeconomic factors. PLoS One 2021;16:e248020.

2. Cotts KG, Cifu AS. Treatment of osteoporosis. JAMA 2018;319:1040-1.

3. Lu PY, Hsieh CF, Tsai YW, Huang WF. Alendronate and raloxifene use related to cardiovascular diseases: differentiation by different dosing regimens of alendronate. Clin Ther 2011;33:1173-9.

4. Rhee CW, Lee J, Oh S, Choi NK, Park BJ. Use of bisphosphonate and risk of atrial fibrillation in older women with osteoporosis. Osteoporos Int 2012;23:247-54.

5. Wilkinson GS, Baillargeon J, Kuo YF, Freeman JL, Goodwin JS. Atrial fibrillation and stroke associated with intravenous bisphosphonate therapy in older patients with cancer. J Clin Oncol 2010;28:4898-905.

6. Pinkerton JV, Thomas S, Dalkin AC. Osteoporosis treatment and prevention for postmenopausal women: current and future therapeutic options. Clin Obstet Gynecol 2013;56:711-21.

7. Erichsen R, Christiansen CF, Froslev T, Jacobsen J, Sorensen HT. Intravenous bisphosphonate therapy and atrial fibrillation/flutter risk in cancer patients: a nationwide cohort study. Br J Cancer 2011;105:8813.

8. Fazmin IT, Huang CL, Jeevaratnam K. Bisphosphonates and atrial fibrillation: revisiting the controversy. Ann N Y Acad Sci 2020;1474:1526.

9. Khosla S, Bilezikian JP, Dempster DW, Lewiecki EM, Miller PD, Neer $\mathrm{RM}$, et al. Benefits and risks of bisphosphonate therapy for osteoporosis. J Clin Endocrinol Metab 2012;97:2272-82.

10. Herrera L, Leal I, Lapi F, Schuemie M, Arcoraci V, Cipriani F, et al. Risk of atrial fibrillation among bisphosphonate users: a multicenter, population-based, Italian study. Osteoporos Int 2015;26:1499-506.

11. Yang HY, Huang JH, Chiu HW, Lin YK, Hsu CY, Chen YJ. Vitamin D and bisphosphonates therapies for osteoporosis are associated with different risks of atrial fibrillation in women: a nationwide populationbased analysis. Medicine (Baltimore) 2018;97:e12947.

12. Bunch TJ, Anderson JL, May HT, Muhlestein JB, Horne BD, Crandall $\mathrm{BG}$, et al. Relation of bisphosphonate therapies and risk of developing atrial fibrillation. Am J Cardiol 2009;103:824-8.

13. Sorensen HT, Christensen S, Mehnert F, Pedersen L, Chapurlat RD, Cummings SR, et al. Use of bisphosphonates among women and risk of atrial fibrillation and flutter: population based case-control study. BMJ 2008;336:813-6.

14. Heckbert SR, Li G, Cummings SR, Smith NL, Psaty BM. Use of alendronate and risk of incident atrial fibrillation in women. Arch Intern Med 2008;168:826-31.

15. Abrahamsen B, Eiken P, Brixen K. Atrial fibrillation in fracture patients treated with oral bisphosphonates. J Intern Med 2009;265:581-92.

16. Huang WF, Tsai YW, Wen YW, Hsiao FY, Kuo KN, Tsai CR. Osteoporosis treatment and atrial fibrillation: alendronate versus raloxifene. Menopause 2010;17:57-63.

17. Grosso A, Douglas I, Hingorani A, MacAllister R, Smeeth L. Oral bisphosphonates and risk of atrial fibrillation and flutter in women: a self-controlled case-series safety analysis. PLoS One 2009;4:e4720.

18. Brandes A, Smit MD, Nguyen BO, Rienstra M, Van Gelder IC. Risk factor management in atrial fibrillation. Arrhythm Electrophysiol Rev 2018;7:118-27.

19. Zulkifly H, Lip GY, Lane DA. Epidemiology of atrial fibrillation. Int J Clin Pract 2018;72:e13070.

20. Kornej J, Borschel CS, Benjamin EJ, Schnabel RB. Epidemiology of atrial fibrillation in the 21st century: novel methods and new insights. Circ Res 2020;127:4-20.

21. Belbasis L, Mavrogiannis MC, Emfietzoglou M, Evangelou E. Environmental factors, serum biomarkers and risk of atrial fibrillation: an exposure-wide umbrella review of meta-analyses. Eur J Epidemiol 2020;35:223-39.

22. Karnik AA, Gopal DM, Ko D, Benjamin EJ, Helm RH. Epidemiology of atrial fibrillation and heart failure: a growing and important problem. Cardiol Clin 2019;37:119-29.

23. Howard PA, Barnes BJ, Vacek JL, Chen W, Lai SM. Impact of bisphosphonates on the risk of atrial fibrillation. Am J Cardiovasc Drugs 2010;10:359-67.

24. Guo Q, Du X, Ma CS. Atrial fibrillation and frailty. J Geriatr Cardiol 2020;17:105-9.

25. Sewerynek E, Stuss M. Bisphosphonates and the risk of atrial fibrillation. Endokrynol Pol 2011;62:93-6.

26. DerSimonian R, Laird N. Meta-analysis in clinical trials revisited. Contemp Clin Trials 2015;45(Pt A):139-45.

27. Sedgwick P. Meta-analyses: what is heterogeneity? BMJ 2015;350: h1435.

28. Stang A. Critical evaluation of the Newcastle-Ottawa scale for the assessment of the quality of nonrandomized studies in meta-analyses. Eur J Epidemiol 2010;25:603-5.

29. Sterne JA, Harbord RM. Funnel plots in meta-analysis. Stata J 2004;4:127-41.

30. Sharma A, Einstein AJ, Vallakati A, Arbab-Zadeh A, Walker MD, Mukherjee D, et al. Risk of atrial fibrillation with use of oral and intravenous bisphosphonates. Am J Cardiol 2014;113:1815-21.

31. Harada M, Van Wagoner DR, Nattel S. Role of inflammation in atrial fibrillation pathophysiology and management. Circ J 2015;79:495-502.

32. Silva PG, Ferreira Junior AE, Teofilo CR, Barbosa MC, Lima Junior RC, Sousa FB, et al. Effect of different doses of zoledronic acid in establishing of bisphosphonate-related osteonecrosis. Arch Oral Biol 2015;60:1237-45.

33. Hewitt RE, Lissina A, Green AE, Slay ES, Price DA, Sewell AK. The bisphosphonate acute phase response: rapid and copious production 
of proinflammatory cytokines by peripheral blood gd T cells in response to aminobisphosphonates is inhibited by statins. Clin Exp Immunol 2005;139:101-11.

34. McClung M, Harris ST, Miller PD, Bauer DC, Davison KS, Dian L, et al. Bisphosphonate therapy for osteoporosis: benefits, risks, and drug holiday. Am J Med 2013;126:13-20.

35. Ylitalo R. Bisphosphonates and atherosclerosis. Gen Pharmacol 2000;35:287-96.

36. Abrahamsen B. Bisphosphonate adverse effects, lessons from large databases. Curr Opin Rheumatol 2010;22:404-9.

37. Sharma A, Chatterjee S, Arbab-Zadeh A, Goyal S, Lichstein E, Ghosh J, et al. Risk of serious atrial fibrillation and stroke with use of bisphosphonates: evidence from a meta-analysis. Chest 2013;144:1311-22.

38. Kim HW, Park CW, Shin YS, Kim YS, Shin SJ, Kim YS, et al. Calcitriol regresses cardiac hypertrophy and QT dispersion in secondary hyperparathyroidism on hemodialysis. Nephron Clin Pract 2006;102:c21-9.

39. van Geel TA, Bliuc D, Geusens PP, Center JR, Dinant GJ, Tran T, et al. Reduced mortality and subsequent fracture risk associated with oral bisphosphonate recommendation in a fracture liaison service setting: a prospective cohort study. PLoS One 2018;13:e0198006.

40. Yatsuya H, Li Y, Hilawe EH, Ota A, Wang C, Chiang C, et al. Global trend in overweight and obesity and its association with cardiovascular disease incidence. Circ J 2014;78:2807-18.

41. Sedgwick P. Bias in observational study designs: case-control studies. BMJ 2015;350:h560.

42. Pandis N. Bias in observational studies. Am J Orthod Dentofacial Orthop 2014;145:542-3. 